\title{
Modelos de regressão não linear aplicados a grupos de acessos de alho
}

\author{
Renata M Reis ${ }^{1}$; Paulo R Cecon ${ }^{1}$; Mário Puiatti ${ }^{1}$; Fernando L Finger ${ }^{1}$; Moysés Nascimento1; Fabyano F \\ Silva'; Antônio PS Carneiro' ${ }^{1}$; Anderson R Silva ${ }^{2}$ \\ 1UFV, 36571-000Viçosa-MG; renatamreis@gmail.com; cecon@ufv.br; mpuiatti@ufv.br; ffinger@ufv.br; moysesnascim@ufv.br; \\ fabyanofonseca@ufv.br; policarpo@ufv.br; ${ }^{2}$ ESALQ, C. Postal 09, 13418-900 Piracicaba-SP; ar.silva@usp.br
}

\section{RESUMO}

O principal objetivo deste estudo foi comparar modelos de regressão não linear aptos a descreverem o acúmulo de massa seca de diferentes partes da planta do alho ao longo do tempo $(60,90,120 \mathrm{e}$ 150 dias após plantio). Objetivou-se também identificar acessos semelhantes em relação às características avaliadas por meio de análises de agrupamento. Foram utilizados 20 acessos de alho pertencentes ao Banco de Germoplasma de Hortaliças da Universidade Federal de Viçosa (BGH/UFV). O teor de massa seca da folha, do pseudocaule, do bulbo e da raiz foram definidos como variáveis na análise de agrupamento (algoritmo de Ward utilizando como medida de dissimilaridade a distância quadrada generalizada de Mahalanobis), que resultou na indicação de um número ótimo (critério de Mojena) de três grupos de acessos, cujas médias de massa seca do bulbo, da raiz e total da planta em cada tempo de interesse foram utilizadas para o ajuste de cinco modelos de regressão não linear (Mitscherlich, Gompertz, Logístico, Brody e von Bertalanffy). A identificação do modelo que melhor se ajustou às três características de cada grupo foi realizada mediante coeficiente de determinação $\left(\mathrm{R}^{2}\right)$, o quadrado médio do resíduo (QMR) e o desvio médio absoluto dos resíduos (DMA). Comparando os valores desses avaliadores observou-se que, para as três caraterísticas dos três grupos, o modelo que melhor se ajustou foi o Logístico.

Palavras-chave: Allium sativum, análise de agrupamento, comparação de modelos.

\begin{abstract}
Nonlinear regression models applied to clusters of garlic accessions

The main objective of this study was to compare nonlinear regression models able to describe the dry matter accumulation in different parts of the garlic plant over time (60, 90, 120 and 150 days after planting). This study also aimed to identify similar accessions with respect to the characteristics evaluated through cluster analysis. We used 20 garlic accessions belonging to the Vegetable Germplasm Bank of Universidade Federal de Viçosa. The dry matter content of leaves, pseudostems, bulbs and roots were defined as variables in the cluster analysis (Ward algorithm using as dissimilarity measure the squared generalized Mahalanobis distance), which resulted in the appointment of an optimal number (Mojena criteria) of three groups of accessions, whose means of dry matter of bulbs, of roots and of the whole plant were used for fitting five nonlinear regression models (Mitscherlich, Gompertz, Logistic, von Bertalanffy and Brody). The identification of the model that best fitted the three characteristics of each group was carried out by coefficient of determination $\left(\mathrm{R}^{2}\right)$, the error mean square and the average deviation absolut error. Comparing the values of these evaluators, we found that, for the three characteristics of the three groups, the best fitted model was the Logistic.
\end{abstract}

Keywords: Allium sativum, clustering analysis, comparison of models.

\section{(Recebido para publicação em 19 de novembro de 2013; aceito em 16 de abril de 2014) (Received on November 19, 2013; accepted on April 16, 2014)}

$\mathrm{O}$ alho (Allium sativum) é uma hortaliça que se destaca na culinária mundial e, particularmente, na brasileira, por possuir características acentuadas de aroma e sabor que lhe atribuem propriedades condimentares e também por suas propriedades medicinais (Menezes Sobrinho et al., 1999). Apresenta ainda importância econômica e possui grande influência no sistema de agricultura familiar, por empregar mão-de-obra desde o plantio até a colheita (Trani, 2009). Em relação à sua diversidade genética existe grande variabilidade genética que apresenta a necessidade de serem conduzidas pesquisas com o objetivo de estudar esta divergência genética.

Na literatura são apresentadas várias técnicas para estudo de divergência genética (Menezes Sobrinho et al., 1999; Faria et al., 2012), dentre os quais se destaca a análise de agrupamentos, que utiliza os valores das variáveis para esquematizar formas de agrupar os objetos em classes, de modo que objetos similares estejam na mesma classe, ou ainda, que tem por finalidade reunir os indivíduos em um determinado número de grupos, de modo que exista grande homogeneidade dentro de cada grupo e heterogeneidade entre eles (Johnson \& Wichern, 2007).
Para que se faça um manejo adequado por meio de informações relevantes e também para que se detectem possíveis problemas no desenvolvimento da cultura do alho recomenda-se estudar a trajetória de crescimento e o acúmulo de massa seca de suas diferentes partes. Para tanto, modelos de regressão não linear são geralmente recomendados. Tais modelos já foram utilizados com sucesso em outros estudos envolvendo bananeiras (Maia et al., 2009), arroz (Agostinetto et al., 2004), milho doce (Araújo et al., 2001) e batata (Silva et al., 2007), entre outros. A vantagem desses modelos é que apresentam pa- 
râmetros com interpretações biológicas como o valor máximo da característica em questão e a velocidade do crescimento. Porém, diante da possibilidade de diferentes modelos, torna-se relevante a comparação da qualidade do ajuste dos mesmos.

Diante dos aspectos considerados e da ausência desse tipo de estudo na literatura para a cultura do alho, este trabalho teve por objetivo comparar modelos de regressão não linear aptos a descreverem o acúmulo de massa seca de diferentes partes da planta do alho ao longo do tempo, como a massa seca do bulbo, massa seca da raiz e massa seca total da planta em diferentes acessos. E, com o intuito de se trabalhar apenas com grupos de acessos semelhantes, avaliou-se a diversidade genética de 20 acessos de alho registrados no Banco de Germoplasma de Hortaliças (BGH/ UFV).

\section{MATERIAL E MÉTODOS}

Os dados foram provenientes de um experimento realizado no período de março a novembro de 2010, em área experimental pertencente ao setor de olericultura do Departamento de Fitotecnia da Universidade Federal de Viçosa (UFV), Viçosa-MG (2045'S, $42^{\circ} 51^{\prime} \mathrm{O}$, altitude de $650 \mathrm{~m}$ ).

$\mathrm{O}$ delineamento experimental foi de blocos completos casualizados com quatro repetições. Foram avaliados 20 acessos de alho registrados no Banco de Germoplasma de Hortaliças (BGH/ UFV), sendo um originário de Capim Branco-MG (cv. Alho Roxão), três de Florestal-MG (cv. Barbado do Rio Grande, Montes Claros e Sacaia de Guanhães), um de Rio Pantojas-ES (cv. Sapé), um de $\mathrm{S}^{\text {ta }}$ Maria, $\mathrm{S}^{\text {ta }}$ Leopoldina-ES [cv. não identificado (1)], um de Vargem Alegre-MG [cv. não identificado (2)], um de Brasília-DF (cv. Cultura de Tecidos), seis de Viçosa-MG [cv. Amarante, Centenário, Cateto Roxo Seleção, Cajuru, Chinês (1) e Chinês (2)], um de Nova Venécia-ES (cv. Cateto Roxo, $\mathrm{d}^{\mathrm{t}}$ arredondado), um de Presidente Médice-RO (cv. Presidente Médice), dois do Espírito Santo [cv. Amarante EPAMIG (1) e Amarante EPAMIG (2)] e dois de origem não identificada (cv. Peruano e Amarante Novo Cruzeiro). A avaliação de massa seca das partes das plantas desses acessos foi realizada em quatro períodos, 60, 90, 120 e 150 dias após plantio.

O teor de massa seca foi quantificado para as seguintes partes da planta: folha (MSF), pseudocaule (MSP), bulbo (MSB), raiz (MSR) e total da planta (MSTP). O procedimento de obtenção destes dados envolveu secagem em estufa, com ventilação forçada a $65^{\circ} \mathrm{C}$, até massa constante, expressa em gramas por planta. A MSTP foi obtida pelo somatório da MSF, MSP, MSB e MSR.

Em uma primeira etapa realizou-se a análise de agrupamento para obter grupos envolvendo acessos de alho similares, na qual as massas secas da folha, do pseudocaule, do bulbo e da raiz foram definidas como as variáveis para o agrupamento. Foi utilizado o método proposto por Ward, que é também conhecido como "Método da Variância Mínima”, pois considera como função objetivo minimizar a soma de quadrados dentro dos grupos. Essa análise foi realizada no software $\mathrm{R}$ versão 2.15.1 ( $\mathrm{R}$ Development Core Team, 2012) com a função hclust do pacote stats, em que a matriz de distâncias foi a de distâncias generalizadas de Mahalanobis.

Para a determinação do número de grupos, utilizou-se o procedimento sugerido por Mojena (1977), que se baseia no tamanho relativo dos níveis de fusões (distâncias) no dendrograma para determinar o ponto de corte dos dendrogramas gerados pelos métodos hierárquicos e, assim, definir o número de grupos. Propõe selecionar o número de grupos no passo j que, primeiramente, satisfizer a inequação $\alpha_{j+1}>\theta_{k}$, em que $\alpha_{j+1}$ é o valor de distância do nível de fusão correspondente ao passo $\mathrm{j}+1$ e $\theta_{k}$ é o valor referencial de corte, dado por $\theta_{k}=\bar{\alpha}+k \hat{\sigma}_{\alpha}$, em que $\bar{\alpha}$ e $\hat{\sigma}_{\alpha}$ são, respectivamente, a média e o desvio padrão dos valores de $\alpha ; k$ é uma constante cujo valor a ser adotado é de $\mathrm{k}=1,25$ como regra de parada na definição do número de grupos. Assim, tem-se que

$$
\begin{aligned}
& \bar{\alpha}=\frac{1}{g-1} \sum_{j=1}^{g-1} \alpha_{j} \mathrm{e} \\
& \hat{\sigma}_{\alpha}=\sqrt{\frac{1}{g-2}\left[\sum_{j=1}^{g-1} \alpha_{j}^{2}-\frac{1}{g-1}\left(\sum_{j=1}^{g-1} \alpha_{j}\right)^{2}\right]}
\end{aligned}
$$

em que $g$ é o número de acessos a serem agrupados. Esses cálculos foram implementados no software R versão 2.15.1 (R Development Core Team, 2012) tendo por base os resultados obtidos da função hclust. Os grupos foram delimitados com o auxílio da função cutree, informando o valor de dissimilaridade obtido com o método de Mojena para definir a altura do "corte" no dendrograma.

Uma vez obtidos os grupos de acessos obteve-se as médias das variáveis em cada tempo. Para descrever a trajetória longitudinal do acúmulo de massa seca do bulbo, da raiz e total da planta foram utilizados os seguintes modelos: Mitscherlich (M),

$y_{i}=\beta_{1}\left[1-e^{\left(\beta_{\mathrm{s}} \beta_{2}-\beta_{\mathrm{s}} x_{i}\right)}\right]+\varepsilon_{i} ;$

Gompertz (G),

$y_{i}=\beta_{1} e^{\left[-\beta_{2} e^{\left(-\beta_{\mathrm{s}} x_{i}\right)}\right]}+\varepsilon_{i}$;

Logístico (L),

$y_{i}=\frac{\beta_{1}}{1+\beta_{2} e^{\left(-\beta_{3} x_{i}\right)}}+\varepsilon_{i}$;

Brody (B),

$y_{i}=\beta_{1}\left[1-\beta_{2} e^{\left(-\beta_{\mathrm{s}} x_{i}\right)}\right]+\varepsilon_{i} ;$

von Bertalanffy (vB),

$y_{i}=\beta_{1}\left[1-\beta_{2} e^{\left(-\beta_{\mathrm{g}} x_{i}\right)}\right]+\varepsilon_{i}$, em que $\beta_{1}$ é o parâmetro que representa o peso assintótico; $\beta_{2}$ é um parâmetro de locação, sem interpretação biológica; $\beta_{3}$ é o parâmetro que representa a taxa de maturidade, ou velocidade do crescimento; $y_{i}$ representa a observação na variável dependente (ou variável resposta); $x_{i}$ representa a variável independente (ou variável preditora); $\varepsilon_{i}$ representa o erro aleatório, pressupondo $\varepsilon_{i} \sim N\left(0, \sigma^{2}\right)$. Esses modelos são bastante utilizados na literatura para descrever curvas de crescimento 
de plantas e também acúmulo de massa seca, como em bananeira (Maia et al., 2009) utilizando os modelos G, L, B e vB; em cebola (Pôrto et al., 2006) utilizando o modelo L; em leucena (Brito et al., 2007) utilizando os modelos M e vB.

Para o ajuste dos modelos utilizou-se a função $n l s$, do pacote stats do software livre R versão 2.15.1 (R Development Core Team, 2012), que tem por base o método dos mínimos quadrados ordinários. O método iterativo utilizado foi o de Gauss-Newton.

A fim de comparar os modelos propostos, utilizou-se os seguintes avaliadores da qualidade do ajuste: coeficiente de determinação $\left(R^{2}\right)$,

$R^{2}=1-\frac{S Q R}{S Q T}$

quadrado médio do resíduo $(Q M R)$, $Q M R=\frac{1}{n-p} \sum_{i=1}^{n}\left(y_{i}-\hat{y}_{i}\right)^{2}$;

e proposto por Sarmento et al. (2006), o desvio médio absoluto dos resíduos (DMA),

$D M A=\frac{1}{n} \sum_{i=1}^{n}\left|y_{i}-\hat{y}_{i}\right|$,

em que $S Q R$ é a soma dos quadrados residuais e $S Q T$ é a soma de quadrados total corrigida pela média, $n$ é o nú- mero de observações, $p$ é o número de parâmetros, $y_{i}$ é o valor observado da massa seca da característica em questão e $\widehat{y}_{i}$ é o valor estimado de massa seca da característica em questão pelo modelo analisado.

\section{RESULTADOS E DISCUSSÃO}

Utilizando o método de Mojena (1977), com $\mathrm{k}=1,25$, determinou-se um ponto de corte no dendrograma obtido com o algoritmo de Ward na distância 16,63 (valor do critério $\theta_{\mathrm{k}}$ do método de Mojena), que corresponde a 40,06\% da distância máxima observada nos níveis de fusão (Figura 1). Assim foram definidos três grupos de acessos. As médias de cada grupo para a massa seca do bulbo (MSB), da raiz (MSR) e total da planta (MSTP) em cada período avaliado estão apresentadas na Tabela 1.

Verifica-se na Tabela 1 que, para a massa seca do bulbo, apesar do grupo I (cvs. Alho Roxão, Montes Claros e Sacaia de Guanhães) ter apresentado as menores médias nos três primeiros períodos avaliados, aos 150 dias após plantio a média dessa característica apresentou-se maior que as médias dos demais grupos. Já para as outras carac- terísticas, as massas secas do grupo I se apresentaram maiores em todos os períodos avaliados durante o crescimento da planta, indicando que os acessos do grupo I, no geral, possuem maiores valores de massa seca das três características (bulbo, raiz e planta toda).

Os dados contidos na Tabela 1 então foram submetidos ao ajuste dos cinco modelos propostos. Para todos os grupos houve convergência de pelo menos dois modelos. Na Tabela 2 são apresentadas as estimativas dos parâmetros e os avaliadores da qualidade do ajuste, de acordo com os modelos ajustados, para as médias de massa seca do bulbo (MSB), da raiz (MSR) e total da planta (MSTP) de cada grupo de acesso.

Pode-se observar na Tabela 2 que os modelos que convergiram para todas as características em todos os grupos foram o modelo de Gompertz (G) e Logístico (L). O modelo de von Bertalanffy (vB) só não convergiu para massa seca do bulbo no grupo I, enquanto o modelo de Brody (B) somente convergiu para massa seca da raiz e massa seca total da planta no grupo III [cvs. Barbado do Rio Grande, não identificado (2), Amarante EPAMIG (1), não identificado (1), Cultura de Tecidos, Centenário, Sapé e Amarante Novo Cruzeiro] e o de Mitscherlich (M) teve convergência

Tabela 1. Média e desvio padrão (em parênteses) da massa seca do bulbo (MSB), da raiz (MSR) e total da planta (MSTP) (g/planta), dos respectivos grupos e dias após plantio [mean and standard deviation (in parentheses) of the dry matter of the bulb (MSB), of the root (MSR) and the whole plant (MSTP) (g/plant), of the respective groups and days after planting). Viçosa, UFV, 2012.

\begin{tabular}{|c|c|c|c|c|c|}
\hline \multirow{2}{*}{ Massa seca } & \multirow{2}{*}{ Grupos* } & \multicolumn{4}{|c|}{ Dias após plantio } \\
\hline & & 60 & 90 & 120 & 150 \\
\hline \multirow{3}{*}{ MSB } & I & $0,089(0,052)$ & $0,495(0,070)$ & $4,671(0,454)$ & $18,550(2,354)$ \\
\hline & II & $0,098(0,035)$ & $0,688(0,130)$ & $6,783(1,605)$ & $14,117(2,341)$ \\
\hline & III & $0,091(0,047)$ & $0,739(0,349)$ & $8,666(2,801)$ & $14,797(1,339)$ \\
\hline \multirow{3}{*}{ MSR } & $\mathrm{I}$ & $0,130(0,026)$ & $0,495(0,102)$ & $1,488(0,150)$ & $2,233(0,404)$ \\
\hline & II & $0,093(0,057)$ & $0,274(0,096)$ & $0,713(0,269)$ & $1,106(0,225)$ \\
\hline & IIII & $0,055(0,032)$ & $0,276(0,085)$ & $1,027(0,451)$ & $1,192(0,277)$ \\
\hline \multirow{3}{*}{ MSTP } & I & $0,996(0,172)$ & $4,558(0,579)$ & $19,083(2,555)$ & $28,258(2,568)$ \\
\hline & II & $0,868(0,211)$ & $4,081(0,661)$ & $17,119(3,912)$ & $20,367(2,959)$ \\
\hline & III & $0,683(0,222)$ & $3,902(0,853)$ & $17,126(3,299)$ & $20,003(1,191)$ \\
\hline
\end{tabular}

*Grupo I é formado pelas cvs. Alho Roxão, Montes Claros e Sacaia de Guanhães; grupo II pelas cvs. Chinês (2), Amarante EPAMIG (2), Cateto Roxo Seleção, Peruano, Cateto Roxo, Amarante, Cajuru, Chinês (1) e Presidente Médice; e grupo III pelas cvs. Barbado do Rio Grande, não identificado (2), Amarante EPAMIG (1), não identificado (1), Cultura de Tecidos, Centenário, Sapé e Amarante Novo Cruzeiro [group I is formed by accessions Alho Roxão, Montes Claros and Sacaia de Guanhães; group II is formed by accessions Chinês (2), Amarante EPAMIG (2), Cateto Roxo Seleção, Peruano, Cateto Roxo, Amarante, Cajuru, Chinês (1) and Presidente Médice; group III is formed by accessions Barbado do Rio Grande, não identificado (2), Amarante EPAMIG (1), não identificado (1), Cultura de Tecidos, Centenário, Sapé and Amarante Novo Cruzeiro]. 
Tabela 2. Estimativas dos parâmetros $\beta_{1}, \beta_{2}$ e $\beta_{3}$, coeficiente de determinação $\left(\mathrm{R}^{2}\right)$, quadrado médio do resíduo (QMR) e desvio médio absoluto dos resíduos (DMA), de acordo com os modelos estudados para as médias de massa seca do bulbo (MSB), da raiz (MSR) e massa seca total da planta (MSTP) de cada grupo de acesso [parameter estimates of the $\beta_{1}, \beta_{2}$ and $\beta_{3}$, coefficient of determination $\left(\mathrm{R}^{2}\right)$, the error mean square (QMR) and the average deviation absolut error (DMA), according to the models studied for the mean of dry matter of the bulb (MSB), of the root (MSR) and the whole plant (MSTP), of each group of accession]. Viçosa, UFV, 2012.

\begin{tabular}{|c|c|c|c|c|c|c|c|c|}
\hline \multirow{2}{*}{$\begin{array}{l}\text { Massa } \\
\text { seca }\end{array}$} & \multirow{2}{*}{ Grupo* } & \multirow{2}{*}{ Modelo** } & \multicolumn{3}{|c|}{ Estimativas } & \multicolumn{3}{|c|}{ Avaliadores*** } \\
\hline & & & $\beta_{1}$ & $\beta_{2}$ & $\beta_{3}$ & $\mathbf{R}^{2}$ & QMR & DMA \\
\hline \multirow{8}{*}{ MSB } & \multirow{2}{*}{ I } & $\mathrm{G}$ & 175,167 & 24,618 & 0,016 & 0,999 & 0,006 & 0,021 \\
\hline & & $\mathrm{L}$ & 26,323 & 69475,178 & 0,080 & 0,999 & 0,002 & 0,013 \\
\hline & \multirow{3}{*}{ II } & $\bar{G}$ & 19,945 & 102,321 & 0,038 & 0,999 & 0,009 & 0,025 \\
\hline & & $\mathrm{L}$ & 15,151 & 99438,967 & 0,094 & 0,999 & 0,003 & 0,016 \\
\hline & & $\mathrm{vB}$ & 33,187 & 3,248 & 0,017 & 0,999 & 0,141 & 0,160 \\
\hline & \multirow{3}{*}{ III } & $\mathrm{G}$ & 17,167 & 306,336 & 0,051 & 0,999 & 0,008 & 0,023 \\
\hline & & $\mathrm{L}$ & 15,234 & 329609,223 & 0,108 & 0,999 & 0,004 & 0,017 \\
\hline & & $\mathrm{vB}$ & 24,000 & 5,112 & 0,024 & 0,994 & 0,831 & 0,404 \\
\hline \multirow{10}{*}{ MSR } & \multirow{3}{*}{ I } & G & 3,100 & 22,042 & 0,028 & 0,997 & 0,009 & 0,040 \\
\hline & & $\mathrm{L}$ & 2,505 & 730,822 & 0,058 & 0,999 & 0,001 & 0,011 \\
\hline & & $\mathrm{vB}$ & 3,795 & 2,411 & 0,018 & 0,995 & 0,017 & 0,056 \\
\hline & \multirow{3}{*}{ II } & G & 2,004 & 10,938 & 0,019 & 0,997 & 0,002 & 0,019 \\
\hline & & $\mathrm{L}$ & 1,345 & 273,042 & 0,048 & 0,999 & 0,001 & 0,007 \\
\hline & & $\mathrm{vB}$ & 3,350 & 1,303 & 0,010 & 0,996 & 0,003 & 0,024 \\
\hline & \multirow{4}{*}{ III } & $\mathrm{G}$ & 1,215 & 1004,136 & 0,072 & 0,997 & 0,003 & 0,014 \\
\hline & & $\mathrm{L}$ & 1,208 & 19044,764 & 0,096 & 0,999 & 0,001 & 0,013 \\
\hline & & B & 8,649 & 1,109 & 0,002 & 0,933 & 0,062 & 0,112 \\
\hline & & $\mathrm{vB}$ & 1,367 & 13,106 & 0,040 & 0,985 & 0,016 & 0,061 \\
\hline \multirow{11}{*}{ MSTP } & \multirow{3}{*}{ I } & $\mathrm{G}$ & 32,904 & 91,689 & 0,043 & 0,998 & 0,941 & 0,264 \\
\hline & & $\mathrm{L}$ & 30,008 & 4472,638 & 0,075 & 0,999 & 0,191 & 0,150 \\
\hline & & $\mathrm{vB}$ & 37,414 & 6,360 & 0,029 & 0,997 & 1,673 & 0,542 \\
\hline & \multirow{3}{*}{ II } & G & 20,861 & 917,014 & 0,070 & 0,998 & 0,753 & 0,217 \\
\hline & & $\mathrm{L}$ & 20,633 & 26094,219 & 0,098 & 0,999 & 0,366 & 0,198 \\
\hline & & $\mathrm{vB}$ & 23,793 & 11,933 & 0,038 & 0,984 & 4,793 & 1,066 \\
\hline & \multirow{5}{*}{ III } & $\mathrm{M}$ & 391,863 & 61,295 & 0,001 & 0,924 & 20,721 & 2,036 \\
\hline & & G & 20,371 & 1428,841 & 0,075 & 0,998 & 0,466 & 0,171 \\
\hline & & L & 20,193 & 46565,013 & 0,104 & 0,999 & 0,224 & 0,150 \\
\hline & & B & 391,857 & 1,039 & 0,001 & 0,924 & 20,721 & 2,036 \\
\hline & & $\mathrm{vB}$ & 23,340 & 12,655 & 0,039 & 0,982 & 5,352 & 1,125 \\
\hline
\end{tabular}

*Grupo I é formado pelas cvs. Alho Roxão, Montes Claros e Sacaia de Guanhães; grupo II pelas cvs. Chinês (2), Amarante EPAMIG (2), Cateto Roxo Seleção, Peruano, Cateto Roxo, Amarante, Cajuru, Chinês (1) e Presidente Médice; grupo III pelas cvs. Barbado do Rio Grande, não identificado (2), Amarante EPAMIG (1), não identificado (1), Cultura de Tecidos, Centenário, Sapé e Amarante Novo Cruzeiro. **Os modelos utilizados são: Mitscherlich (M), Gompertz (G), Logístico (L), Brody (B) e von Bertalanffy (vB). ***Valores em negrito são os que representam os melhores valores para cada avaliador [group I is formed by accessions Alho Roxão, Montes Claros and Sacaia de Guanhães; group II is formed by accessions Chinês (2), Amarante EPAMIG (2), Cateto Roxo Seleção, Peruano, Cateto Roxo, Amarante, Cajuru, Chinês (1) and Presidente Médice; group III is formed by accessions Barbado do Rio Grande, unidentified (2), Amarante EPAMIG (1), unidentified (1), Cultura de Tecidos, Centenário, Sapé and Amarante Novo Cruzeiro. The models used are: Mitscherlich (M), Gompertz (G), Logístico (L), Brody (B) and von Bertalanffy (vB). Values in bold are those that represent the best values for each evaluator].

somente para massa seca total da planta também no grupo III.

Nota-se ainda que os valores para o coeficiente de determinação $R^{2}$ foram bem próximos de 1 , indicando que os modelos foram bem ajustados aos dados dos três grupos para as três massas secas. Para a massa seca do bulbo, no grupo I e no grupo II [cvs. Chinês (2), Amarante EPAMIG (2), Cateto Roxo
Seleção, Peruano, Cateto Roxo, Amarante, Cajuru, Chinês (1) e Presidente Médice], o valor de $R^{2}$ foi o mesmo para os modelos ajustados nesses grupos, já no grupo III o $R^{2}$ mostrou-se menor para 


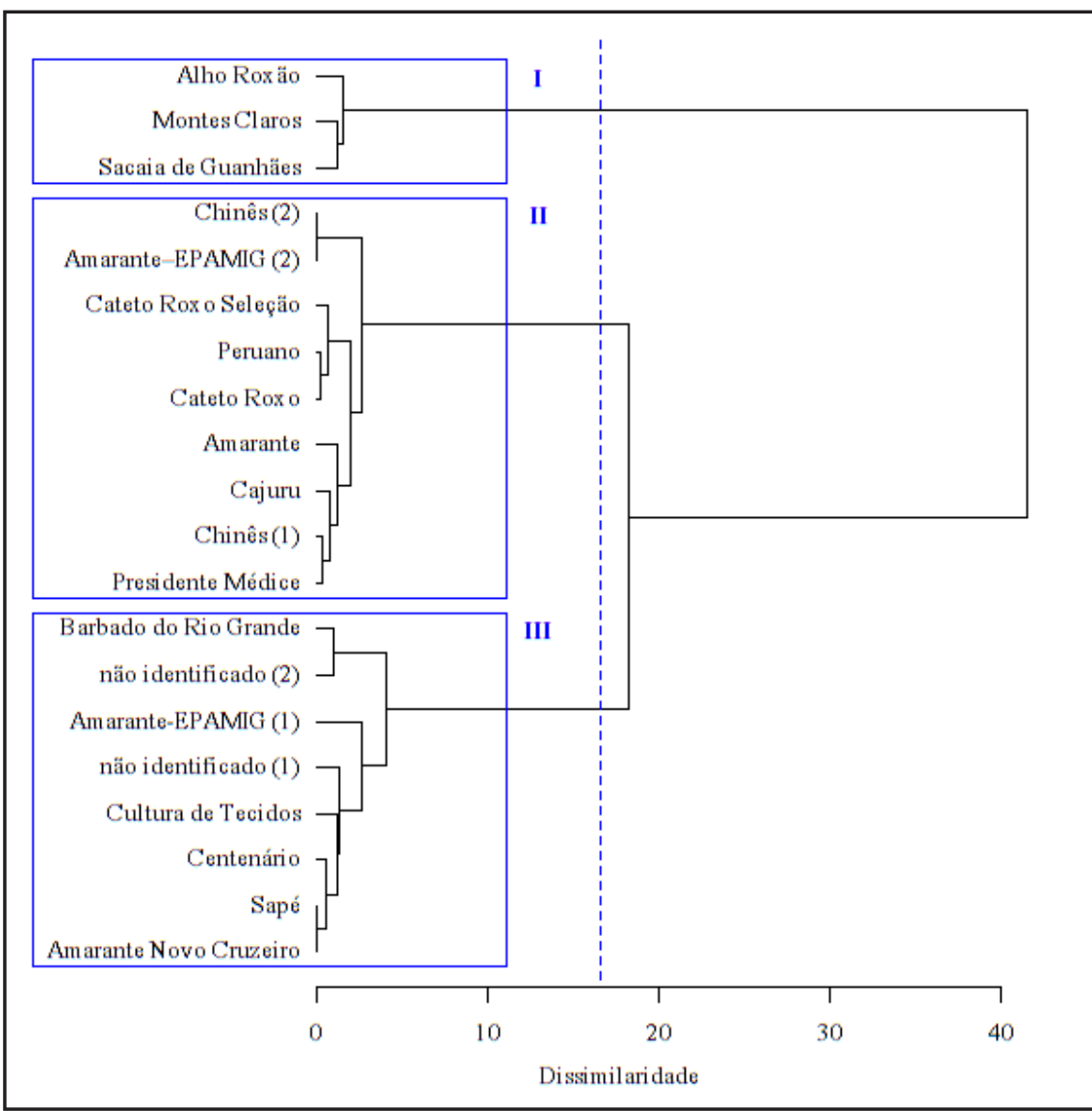

Figura 1. Dendrograma obtido com o algoritmo de Ward com base na distância generalizada de Mahalanobis $\left(\mathrm{D}^{2}\right)$ para agrupamento de 20 acessos de alho pertencentes ao BGH/UFV (dendrogram obtained with Ward's algorithm based on the Mahalanobis distance $\left(\mathrm{D}^{2}\right)$ for cluster analysis of the 20 garlic accessions belonging to BGH/UFV]. Viçosa, UFV, 2012.

o modelo de von Bertalanffy do que para os modelos de Gompertz e Logístico, que tiveram os mesmos valores. Já para a massa seca da raiz e para a massa seca total da planta o modelo que obteve os melhores coeficientes de determinação nos três grupos foi o Logístico.

Observa-se também que para as três características, massa seca do bulbo, da raiz e total da planta, o modelo que apresentou os menores valores de quadrado médio do resíduo (QMR) e de desvio médio absoluto dos resíduos (DMA) foi também o Logístico nos três grupos, sendo assim o modelo que melhor descreve as três características nos três grupos.

A Figura 2 apresenta as curvas do modelo Logístico (modelo escolhido como o de melhor ajuste) para cada grupo de acessos em relação às massas secas do bulbo, da raiz e total da planta. Pode-se verificar na Figura 2(a) que a et al., 2009).

As informações obtidas na Figura $2 \mathrm{~b}$ condizem com os valores observados na Tabela 1. Essa tabela mostra que as maiores médias de massa seca da raiz foram apresentadas pelo grupo I e isto é verificado na Figura $2 \mathrm{~b}$ quando se observa que a curva contínua, referente ao grupo I, está sempre acima das demais curvas, representantes dos outros dois grupos de acessos, lembrando que o modelo Logístico foi o melhor ajustado também para essa característica. Verifica-se que o grupo II já atingiu seu peso assintótico por volta dos 130 dias após plantio, enquanto os grupos I e III não atingiram o peso assintótico dentro do intervalo de 150 dias após plantio.

Observa-se na Figura 2c que as curvas de modelo Logístico representantes dos grupos II e III para a massa seca total da planta foram bem semelhantes. Já o grupo I, que teve médias maiores em relação aos outros dois grupos, como foi verificado na Tabela 1, apresentou também a curva do modelo Logístico acima das demais curvas. Além disso, verifica-se que as curvas dos grupos II e III atingiram seu peso assintótico por volta dos 130 dias após plantio, enquanto a curva o grupo I não atinge $o$ peso assintótico durante o intervalo de 150 dias após plantio.

No trabalho de Maia et al. (2009) verificou-se também que o modelo Logístico foi o melhor ajustado para descrever curvas de crescimento em bananeira, Pôrto et al. (2006), utilizaram este mesmo modelo para descrever acúmulos de massa seca da parte aérea, do bulbo e da planta inteira da cultura de cebola, tendo como resultados ótimos ajustes.

Assim, conclui-se que o modelo Logístico apresentou os melhores ajustes para descrever o acúmulo de massa seca do bulbo, da raiz e total da planta de três grupos de acessos de alho pertencentes ao Banco de Germoplasma de Hortaliças da UFV, grupos estes divergentes principalmente em relação ao acúmulo de massa seca no estádio fenológico final.

\section{AGRADECIMENTOS}

Os autores agradecem à Coorde- 


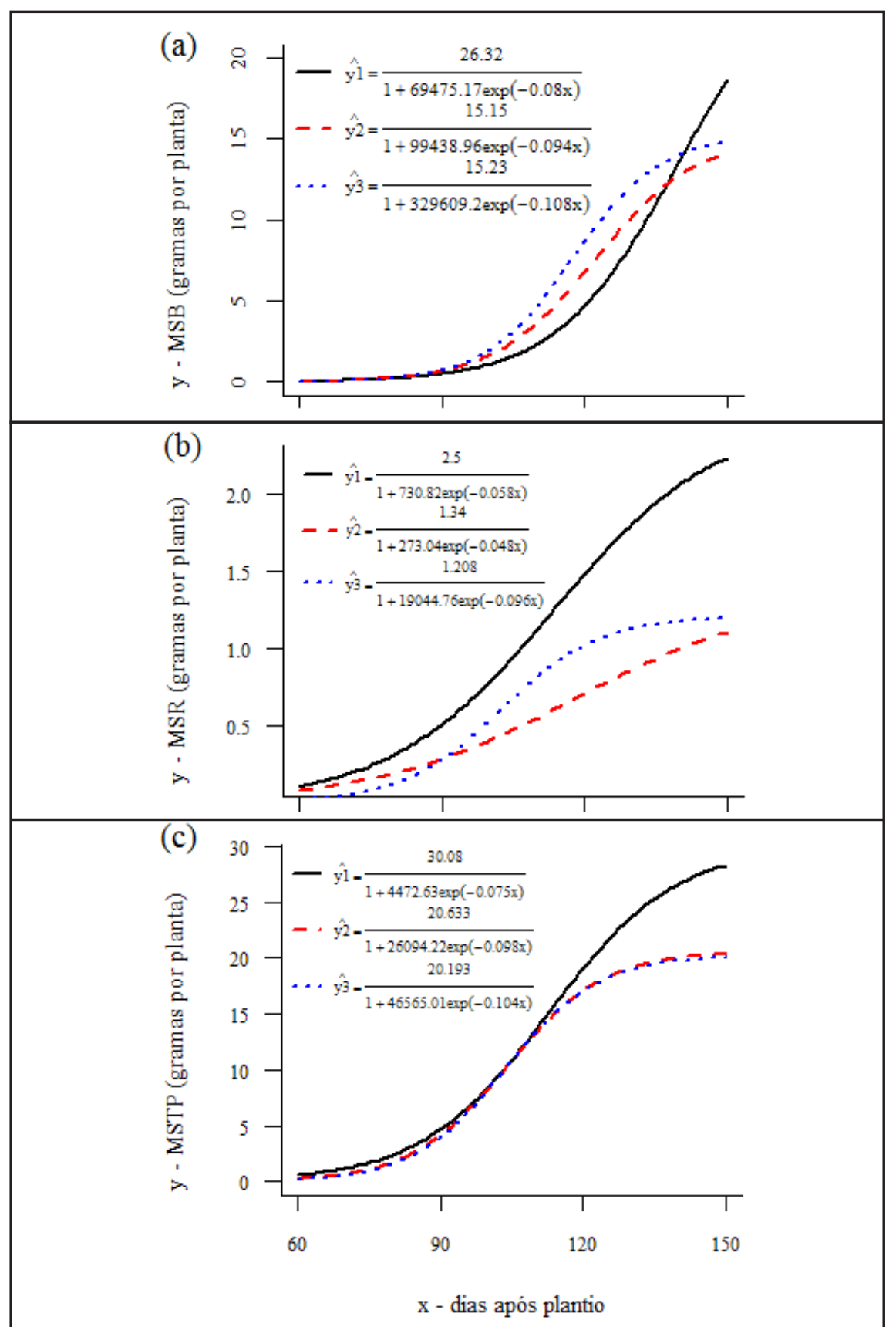

Figura 2. Curvas de acúmulo de massa seca do bulbo (a), da raiz (b) e total da planta (c) ajustadas segundo o modelo Logístico de cada grupo, em que o grupo I é formado pelas cvs. Alho Roxão, Montes Claros e Sacaia de Guanhães; grupo II pelas cvs. Chinês (2), Amarante EPAMIG (2), Cateto Roxo Seleção, Peruano, Cateto Roxo, Amarante, Cajuru, Chinês (1) e Presidente Médice; e grupo III pelas cvs. Barbado do Rio Grande, não identificado (2), Amarante EPAMIG (1), não identificado (1), Cultura de Tecidos, Centenário, Sapé e Amarante Novo Cruzeiro [curves of dry matter accumulation of the bulb (a), of the root (b) and the whole plant (c) fitted according to Logistic model of each group, in which the group I is formed by accessions Alho Roxão, Montes Claros and Sacaia de Guanhães; group II is formed by accessions Chinês (2), Amarante EPAMIG (2), Cateto Roxo Seleção, Peruano, Cateto Roxo, Amarante, Cajuru, Chinês (1) and Presidente Médice; group III is formed by accessions Barbado do Rio Grande, não identificado (2), Amarante EPAMIG (1), não identificado (1), Cultura de Tecidos, Centenário, Sapé and Amarante Novo Cruzeiro]. Viçosa, UFV, 2012.

nação de Aperfeiçoamento de Pessoal de Nível Superior (CAPES) e ao Conselho Nacional de Desenvolvimento
Fundação de Amparo a Pesquisa do Estado de Minas Gerais (FAPEMIG) pelo financiamento do projeto de pesquisa ao qual este trabalho está vinculado.

\section{REFERENNCIAS}

AGOSTINETTO D; FLECK NG; RIZZARDI MA; BIANCHI MA; SILVA PRF. 2004. Comparação de modelos matemáticos na estimativa das perdas de produtividade de grãos em arroz irrigado. Pesquisa Agropecuária Brasileira 39: 847-856.

ARAÚJO EF; CORRÊA PC; SILVA RF. 2001. Comparação de modelos matemáticos para descrição das curvas de dessorção de sementes de milho-doce. Pesquisa Agropecuária Brasileira 36: 991-995.

BRITO CCR; SILVA JAA; FERREIRA RLC; SANTOS ES; FERRAZ I. 2007. Modelos de crescimento resultantes da combinação e variações dos modelos de Chapman-Richards e Silva-Bailey aplicados em Leucaena leucocephala. Ciência Florestal 17: 175-185.

FARIA PN; CECON PR; SILVA AR; FINGER FL; SILVA FF; CRUZ CD; SÁVIO FL. 2012. Métodos de agrupamento em estudo de divergência genética de pimentas. Horticultura Brasileira 30: 428-432.

JOHNSON RA; WICHERN DW. 2007. Applied multivariate statistical analysis. New Jersey, USA: Pearson Prentice Hall. 773p.

MAIA E; SIQUEIRA DL; SILVA FF; PETERNELLI LA; SALOMÃO LCC. 2009. Método de comparação de modelos de regressão não-lineares em bananeiras. Ciência Rural 39: 1380-1386.

MENEZES SOBRINHO JA; CHARCHAR JM; ARAGÃO FAS. 1999. Caracterização morfológica de germoplasma de alho por análises multivariada, componentes principais e variáveis canônicas. Horticultura Brasileira 17: 96-101.

MOJENA R. 1977. Hierarquical grouping method and stopping rules: an evaluation. Computer Journal 20: 359-363.

PÔRTO DRQ; CECÍLIO FILHO AB; MAY A; BARBOSA JC. 2006. Acúmulo de macronutrientes pela cebola 'Optima' estabelecida por semeadura direta. Horticultura Brasileira 24: 470-475.

R DEVELOPMENT CORE TEAM. 2012. $R$ : A language and environment for statistical computing: R Foundation for Statistical Computing version 2.15.1 (software). Austria.

SARMENTO JLR; REGAZZI AJ; SOUZA WH; TORRES RA; BREDA FC; MENEZES GRO. 2006. Estudo da curva de crescimento de ovinos Santa Inês. Revista Brasileira de Zootecnia 35: 435-442.

SILVA MCC; FONTES PCR; MIRANDA GV. 2007. Modelos estatísticos para descrever a produtividade de batata em função da adubação nitrogenada. Horticultura Brasileira 25: 360-364.

TRANI PE. 2009. Cultura do alho (Allium sativum): Diagnóstico e recomendações para seu cultivo no Estado de São Paulo. Artigo em Hypertexto. Disponível em: http://www. infobibos.com/Artigos/2009_2/alho/index. htm. Acessado em: 29 de junho de 2012. 